\title{
Distinctive features of the morphometric parameters of the heart of the Amur tiger (Panthera tigris altaica) in natural habitat and in captivity
}

\author{
R.A. Zhilin ${ }^{1}$, I.P. Korotkova ${ }^{1}$, E.N. Lyubchenko ${ }^{1}$, A.A. Kozhushko, ${ }^{1,}$, and D.V. Kapralov ${ }^{1}$
}

\begin{abstract}
In the process of working with anatomical material supplied to the Animal Disease Diagnostic Center of the Primorskaya State Agricultural Academy, in the order of forensic examinations, a characteristic feature consisting in differences in the structure of the organs of animals living in natural habitat and in captivity was revealed. Evolutionary forces are reflected in the adaptability of animals to survive in the wild, honing adaptive characteristics and sweeping away all unnecessary things. Studying previously published works on the topic in question, it can be noted that the heart of animals in their natural habitat is slender, with a strong left side and relatively weak right side. As a rule, trabecular structures in such animals are smoothed, maximally "built" into the walls of the heart chambers. It is not often possible to find crossbeams as an element of myoendocardial formations in wild animals. However, for the internal structures of the human heart and domesticated animal species, this is not uncommon. There is an opinion that additional muscle-trabecular elements, such as: muscle crossbeams [3]; additional papillary muscles; pectinate muscles of the atria, located in a storey network - take part in the intensification of heart contractions and the creation of a swirling blood flow, providing its translational-rotational movement. This process can be considered an adaptive response to a decrease in physical activity during the evolution of a species, formed from birth to death of an organism. In the course of postmortem examinations, we examined the heart of a four-year-old female Amur tiger raised in captivity with the distinctive features of myoendocardial formations in comparison with other individuals of this species.
\end{abstract}

\section{Introduction}

The heart, being the central organ of the mammalian cardiovascular system, is naturally an object of study by many morphological researchers. Many parameters of morphometry and heart activity have been identified and described $[4,5,6,7]$. However, many species have not yet been sufficiently studied from the morphological point of view.

Also, some authors in their works note the distinctive features in the structure of wild and domestic animals of different species, domesticated and living in the natural environment

\footnotetext{
${ }^{*}$ Corresponding author: Shurban.12@mail.ru
} 
representatives of the same species [4,7].

In our work, we study the corpses of wild animals, but there are also individuals of the same species, but kept in the zoos. In December 2014, an adult four-year-old female Amur tiger, which died due to mechanical asphyxia, was opened. The animal was not removed from its natural habitat, but from childhood it was kept in the zoo's cage, since it was born from the same tigress, who spent all her life in captivity.

Having available data on the morphometric parameters of the heart of representatives of the Panthera tigris altaica subspecies [1], we were able to carry out a comparative analysis of this organ in animals in different habitats. It should be noted that the systematized data we have on the anatomy of the heart of wild tigers belong to the group of adult males. The female is inferior to them both in body weight and in organ weight. Therefore, all our comparisons are in percentage terms.

The purpose of our study is to give a comparative characteristic of the morphometric parameters of the heart of the Amur tiger in different habitats.

\section{Materials and methods}

To compare the features of the formation of the internal structures of the heart, we studied the heart of a female Amur tiger, which had been kept in the zoo all her life. The animal's corpse was presented for autopsy to the Animal Disease Diagnostic Center of the Primorskaya State Agricultural Academy.

The heart was removed according to the instructions for collection and preparation [2]. Photo and video recordings were carried out using a digital camera. The morphometry of the heart structures was performed using a flexible metal ruler and a caliper with a measurement step of $0.01 \mathrm{~cm}$. The age of the animal was determined from the words of the zoo staff. The parameters and structural features of the structures of the organ are compared with those of a group of adult animals that lived in the wild.

After extraction, isolation and purification of blood residues, the heart was subjected to linear measurements.

\section{Research results}

The data of the following morphological components of the heart was presented: atrioventricular valve cusps, tendinous cords, main and additional papillary muscles, elements of the trabecular network of the ventricles; pectinate muscles of the atria.

The heart index was $73.73 \%$ and corresponds to an elliptical shape, which corresponds to the norms in adult Amur tigers [1]. The relative mass of the organ is $0.38 \%$. The same indicator in the group of wild adults was $0.42 \%$.

The wall thickness of the left ventricle of the Amur tiger in natural habitat is $26.21 \pm 1.77$; right $-10.16 \pm 0.76 \mathrm{~mm}$. In an animal in captivity, these indicators were 12.1 and $4.03 \mathrm{~mm}$, respectively. The ratio of the thickness of the ventricles in tigers in natural habitat was approximately $2.5 / 1$. In an animal in the zoo, this figure is $3 / 1$.

The thickness of the wall of the right atrium prevailed over the same indicator of the left atrium in animals of both habitats. In animals in the wild, the wall thickness of the right atrium is $4.21 \pm 0.60$ and the left one is $3.43 \pm 0.16 \mathrm{~mm}$. In a zoo individual, these indicators are 1.43 and $1.35 \mathrm{~mm}$, respectively.

When studying the internal structures of the atria of the studied individual, we found a striking feature - the structure of the pectinate muscles. On the surface of the right atrial appendage, they are multiple, located in different directions and form a dense 2-3-storey network. The number of pectinate muscles of the 1 st order is 5, of the 2 nd order - 16 units, 
which in general corresponds to the numerical indicators in wild Amur tigers [1]. The muscles of the peripheral layer are detached from the wall in the median part (Fig. 1).

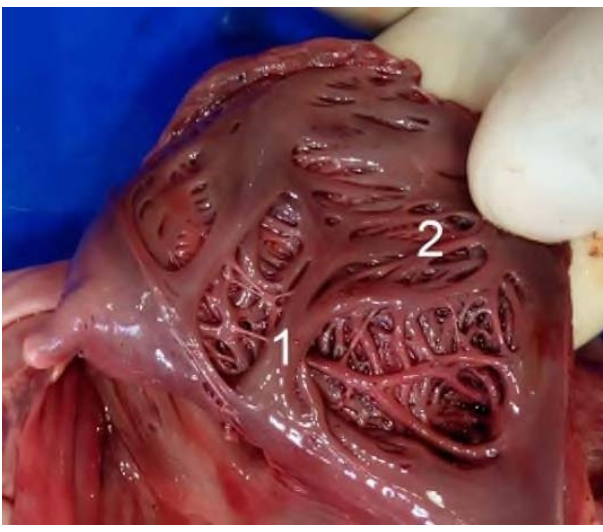

A

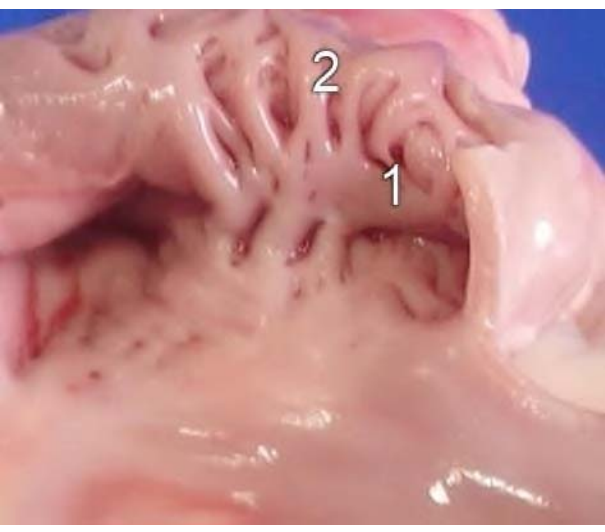

B

Fig. 1. The inner surface of the right atrial appendage: A - an Amur tiger kept in captivity; B - an Amur tiger in natural habitat. 1 - pectinate muscles of the first order; 2 - pectinate muscles of the second order.

The area of the inner surface of the left atrium is less than that of the right one. This feature can be traced in animals from natural habitat and in a zoo individual. In the left atrium of the heart of a female tiger kept in captivity, no freely located crossed pectinate muscles were found, as well as in the right one. There are 5 units of muscles of the $1^{\text {st }}$ order and 9 of the $2^{\text {nd }}$ order. These numerical indicators fit into the species norms [1]

The internal architectonics of the ventricles is more complex, it includes: fibrous rings of the corresponding atrioventricular valves, with cusps elastically attached to them; elements of the trabecular-papillary apparatus (septomarginal and fleshy trabeculae, papillary muscles, tendinous cords).

Variability of the internal structures of the ventricles. The intracavitary parietal formations that we encountered in the process of working with the hearts of Amur tigers from natural habitat are distinguished by smooth shapes. Fleshy trabeculae adjoin the walls of the ventricles. This feature manifests itself in animals with age; in young animals, the myoendocardial relief is more pronounced [1].

Studying the heart of an individual from the zoo, we encountered such formations as muscle crossbeams - formations that, both in the left and in the right ventricle, carry out a parietal connection between points located at various distances from each other on the trabecular myocardium, as well as between them and the fibrous rings valves, located at the same distance from each other [3].

Muscular crossbeams are especially well expressed in the right ventricle of the examined individual. These are large, rounded or oval cross-section cushions located parallel to the longitudinal axis of the heart, connecting the fibrous ring of the corresponding atrioventricular valve with the ventricular wall.

There are three main papillary muscles: large, small and hyparterial. Dimensional data were distributed as follows: the largest - small (24.0 in length and $4.02 \mathrm{~mm}$ in diameter), large $(23.03$ by $6.15 \mathrm{~mm})$, and the smallest dimensions - hyparterial $(14.65$ by $6.6 \mathrm{~mm})$. While in wild Amur tigers, the typical size range is distributed as follows: large, small, hyparterial. The number of additional papillary muscles in the right ventricle is also noteworthy. There are six of them, they all belong to the medial wall. The main papillary muscles have a cylindrical shape, additional muscles are cone-shaped (Fig. 2). 


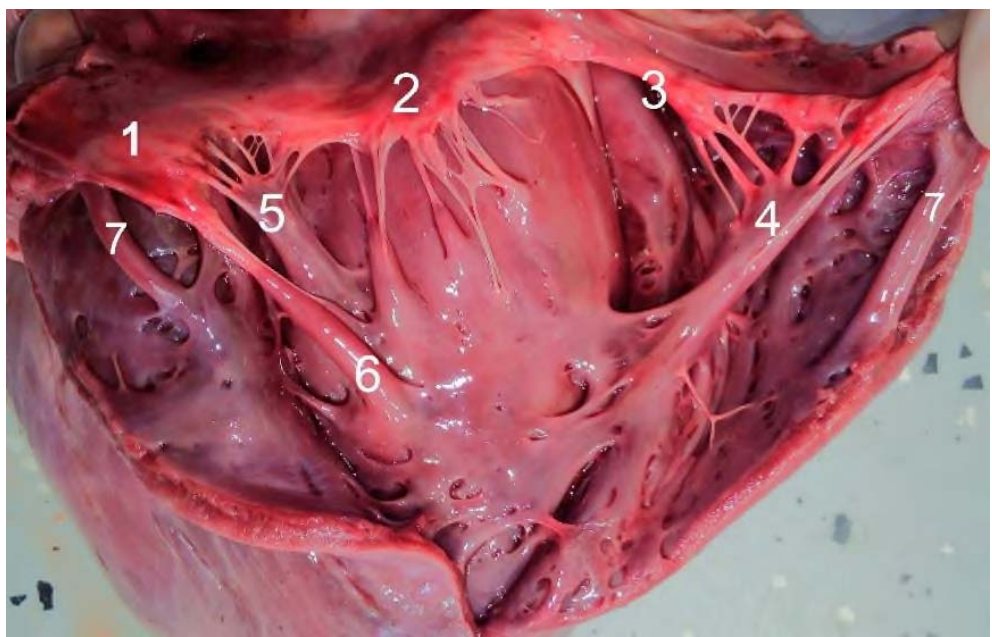

Fig. 2. Myoendocardial formations of the right ventricle of a female Amur tiger kept in a zoo: 1 parietal cusp; 2 - septal cusp; 3 - corner cusp; 4 - large papillary muscle; 5 - small papillary muscle; 6 - additional septal papillary muscle; 7 - muscle crossbeams.

The architectonics of the left ventricle of a zoo individual also has its own outstanding features. Myoendocardial formations, in particular fleshy trabeculae, differ in the severity of the structure, while in wild individuals, they are smoothed. Besides, their number exceeds the average, so: the cranial wall has the crossbeams - 9, crosspieces - 12; caudal - 8 and 10; medial - 7 and 7 units, respectively. What data can we observe in wild individuals regarding these structures? These are: the cranial wall - 5 and 3-4; caudal - 4-5 and 3; medial - 6 and 5 units, respectively [1].

Septomarginal trabeculae are represented by thin tendinous chords (cranial - 19.0 by 1.32 ; caudal -21.75 by $1.4 \mathrm{~mm}$ ) between the bases of the main papillary muscles of the ventricle and the septum lining, repeatedly branching at the points of attachment. In this respect, the studied individual does not differ from its relatives from the wild.

There are two main papillary muscles of the left ventricle. Statistically, the subatrial papillary muscle is cylindrical in shape $-67 \%$, and $33 \%$ belongs to a complex indefinite shape in the animals we studied earlier. There is the same percentage distribution of forms in the second main papillary muscle - the cushion muscle. That is, we can say that the cylindrical shape prevails in these formations. In the zoo animal that we studied, both of the main papillary muscles are powerful, have a complex indefinite shape, the subatrial muscle has two heads, and the cushion muscle - three.

In the left ventricle of the Amur tiger, two main papillary muscles are standardly distinguished, these formations are large, with a wide base. They carry the entire load of preventing everting of the valve cusps by means of many tendinous cords located between their inner surface and the heads of the papillary muscles. Unlike the right ventricle, in which additional papillary muscles are not uncommon, in the left one, we rarely found them in wild individuals in the process of working with material. However, when studying myoendocardial formations in a captive animal, we identified a group of additional papillary muscles. These are relatively small muscles located between the base of the septal cusp and the subatrial papillary muscle and the base of the septal cusp and the cushion papillary muscle (Fig. 3). 


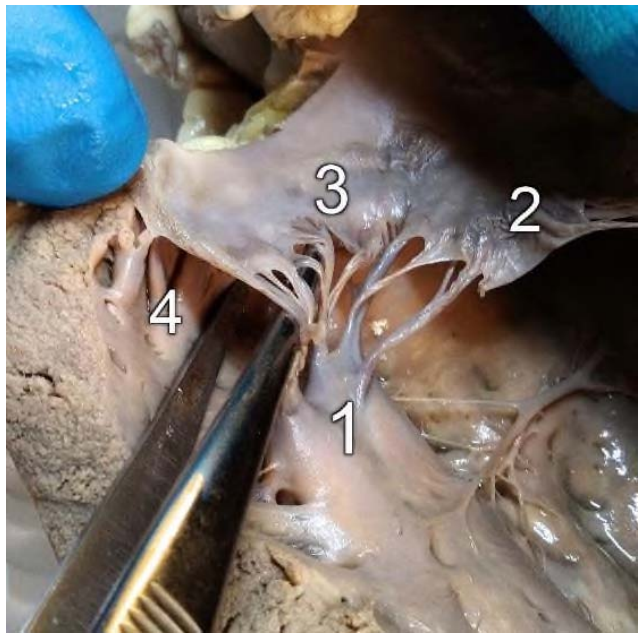

A

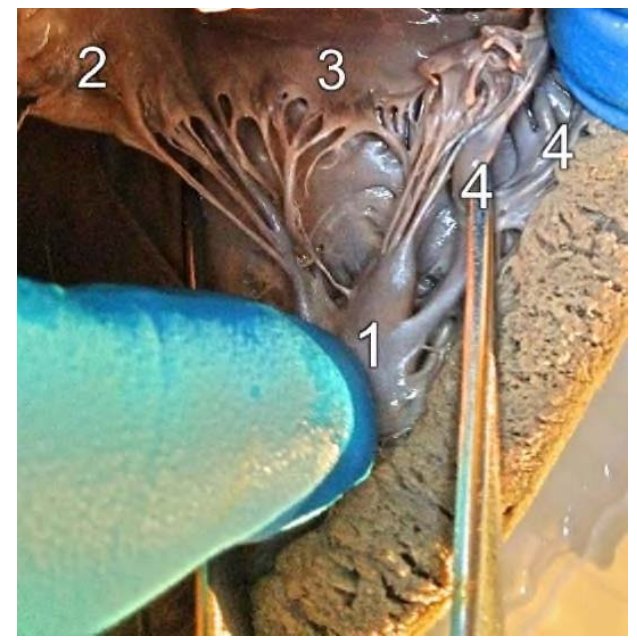

B

Fig. 3. Additional papillary muscles of the left atrioventricular valve: A - complex "septal cusp subatrial papillary muscle"; B - complex "wall cusp - cushion papillary muscle". 1 - the main papillary muscles; 2 - inter-valve commissure; 3 - valve cusps; 4 - additional papillary muscles.

A comparative analysis of the presence of these formations in animals of the same species, but living in different conditions, suggests that their presence in an individual with low physical activity and their absence in wild animals is a consequence of adaptive changes.

\section{Conclusions}

Thus, the following results can be derived from the conducted studies:

1. The heart index of the Amur tiger corresponds to an elliptical shape, which is typical for individuals in natural habitat and in captivity [1].

2. The relative heart mass in an individual in captivity is lower than that of wild relatives. The ratio of the thickness of the ventricles in tigers in natural habitat was approximately 2.5/1. In a zoo animal, this figure was $3 / 1$, which indicates a weaker right ventricle.

3. In zoo animals, myoendocardial formations of the heart chambers (pectinate muscles of the atria, fleshy trabeculae) are well expressed and have the form of networks. In animals from natural habitat, these elements are smoothed out. The papillary-trabecular complex together with the muscular beams form a swirling blood flow, which provides translationalrotational movement [4].

4. The elements of the trabecular network were revealed - the muscular crossbeams, common for humans [3] and unique for the Amur tiger. This fact, obviously, is explained by lower physical activity than in animals living in natural habitats.

5. The number of papillary muscles in the Amur tiger is variable. Besides the main ones, additional ones are found. In the composition of the right atrioventricular valve, they are almost always found. However, in the left one, we've not met them before. The heart of a zoo individual contained a significant number of additional papillary muscles.

6. Significant differences in the architectonics of the internal structures of the atria and ventricles are explained by the belonging of the studied Amur tigers to different ecological groups. This factor is influenced by physical activity, climatic conditions and food supply. 


\section{References}

1. R.A. Zhilin, Morphological parameters of the heart of wild cats in Primorsky Krai: thesis for a Ph.D in Veterinary (Ulan-Ude, 2017)

2. R.A. Zhilin, Morphological parameters of the heart of wild cats of Primorsky Krai. Abstract of a thesis for a Ph.D in Veterinary (Ulan-Ude, 2017)

3. A.P. Stepanchuk, Vestnik of problems of biology and medicine - Poltava 3-2 (95), 174 - 178 (2012)

4. R.Sh. Taiguzin, Age and comparative morphology of the internal structures of the mammalian heart. Abstract of a thesis for a Doctor of Biology (Omsk, 1998)

5. V.N. Tarasevich, N.I. Ryadinskaya, Morphology Journal 153(2-3), 208 (2020)

6. V.N. Tarasevich, N.I. Ryadinskaya, Journal Hippology and Veterinary Medicine 3(23), 178-183 (2020)

7. E.N. Chirkova, Morphology of the heart and its internal structures of mammals of different ecological groups: thesis for a Ph.D. in Biology (Orenburg, 2009) 